inflammation in the pathogenesis of disease. NZM2410 lupus prone mice, expressing a truncated ER functional knockout, survived longer and had significantly reduced renal disease. Yet, a complete knockout of ER was not protective, suggesting the truncated isoform may be protective with the full-length gene necessary for disease. These findings underscore the importance of defining the role of ER in lupus.

Methods While the hormonal impact of estrogen and ER is well studied, less is known about the transcriptional impact of ER in immunity. Our goal is to identify the molecular mechanisms utilized by liganded ER in regulating the inflammatory milieu. To accomplish this, we have identified ER associated target genes, transcription factors and genes involved in the immune response, histone acetylation, and immune related signaling pathways, and determined differences in expression. B cells isolated from 15 female African American lupus patients and 8 age and race matched healthy controls were used for the analysis. In an effort to further understand the molecular mechanisms behind ER function, we examined the transcriptional effects of ER on the inflammatory response. Transient transfections of full length ER ( $66 \mathrm{kDa})$ and a shortened isoform ER46 (lacking the AF-1 activation domain and similar to the truncated ER functional knockout) were initially performed in the human breast cancer cell line MDA-MB-231, which lacks ER.

Results RNA-seq analysis indicates that $64 \%$ of ER associated target genes were differentially expressed between the two groups. The majority of these genes were upregulated in lupus patients compared to controls. Genes with increased expression included TLRs, NFB related transcription factors and IL1. Preliminary results from the transfection experiments indicate that both ER isoforms reduce mRNA expression of the inflammatory cytokines IL-6 and IL-1 twenty-four hours after stimulation with a TLR4 agonist. A decrease in cytokine expression was observed when the short isoform ER46 was overexpressed in relation to ER66. Additional transfections will be carried out in immune relevant cells such as B cells, monocytes, macrophages or dendritic cells.

Conclusions These results support a role for ER in the pathogenesis of SLE via regulation of inflammatory mediators. Future goals include utilizing high throughput sequencing technology to examine the transcriptional impact of ER in monocytes of African American pediatric lupus patients.

Funding Source(s): None

\section{ASSOCIATED FACTORS TO THE PRESENCE OF SERIOUS INFECTIONS IN A LARGE COHORT OF JUVENILE-ONSET SYSTEMIC LUPUS ERYTHEMATOSUS}

\footnotetext{
${ }^{1}$ Jose Maria Pego Reigosa*, ${ }^{2}$ Vicenç Torrente-Segarra, ${ }^{1}$ Victor Del Campo Perez, ${ }^{3}$ Tarek Salman-Monte, ${ }^{4}$ Javier Narváez-García, ${ }^{5}$ Francisco Javier López-Longo, ${ }^{6} J u a n$ Ovalles, ${ }^{7}$ Paula Rubio, ${ }^{8}$ Maria Galindo Izquierdo, ${ }^{9}$ José L Andréu-Sánchez, ${ }^{10}$ Jaime Calvo Alen, ${ }^{11}$ Esther Uriarte, ${ }^{12}$ EvaG Tomero-Muriel, ${ }^{13}$ Ángela Pecondón-Español, ${ }^{14}$ Mercedes FreireGonzález, ${ }^{15}$ Victor Martínez Taboada, ${ }^{16}$ Mireia Moreno-Martínez-Losa, ${ }^{17}$ Mónica IbáñezBarcelo, ${ }^{18}$ Ana Lois-Iglesias, ${ }^{19}$ Ana Sánchez-Atrio, ${ }^{20}$ Loreto Horcada, ${ }^{21}$ Iñigo Rua-Figueroa. ${ }^{1}$ Complexo hospitalario Universitario Vigo; ${ }^{2}$ Rheumatologist; ${ }^{3}$ Parc de Salut Mar; ${ }^{4}$ Hospital de Bellvitge; ${ }^{5}$ Hospital General Universitario Gregorio Marañón; ${ }^{6}$ Hospital Gregorio Marañón; ${ }^{7}$ Hospital Germans Trias I Pujol; ${ }^{8}$ Hospital Universitario 12 De Octubre; ${ }^{9}$ Hospital Puerta de Hierro; ${ }^{10}$ Hospital Universitario Araba; ${ }^{11}$ Hospital Donosti; ${ }^{12}$ Hospital de la Princesa; ${ }^{13}$ Hospital Miguel Servet; ${ }^{14}$ Hospital Juan Canalejo; ${ }^{15}$ Hospital Marqués de Valdecilla; ${ }^{16}$ Parc Tauli; ${ }^{17}$ Son LLatzer; ${ }^{18}$ INIBIC-CHUAC; ${ }^{19}$ Hospital Príncipe de Asturias; ${ }^{20}$ Complejo Hospitalario de Navarra; ${ }^{21}$ Hospital Dr Negrín
}

Background Serious infections are included as one of the main causes of mortality in juvenile-onset systemic lupus erythematosus (jSLE) patients (Torrente-Segarra et al1) and a predictor of poor prognosis in SLE. We aimed to assess the incidence of serious infection and investigate the associated factors and clinical impact in a large jSLE retrospective cohort.

Methods All patients in the Spanish Rheumatology Society Lupus Registry (RELESSER) who meet 4 ACR-97 SLE criteria with disease onset before the age of 18 (jSLE) (Rúa-Figueroa et al2), were retrospectively investigated for serious infections (defined as either the need for hospitalization with parenteral antibiotherapy for a potentially fatal infection or death caused by the infection). Patients with and without infections were compared in terms of jSLE severity, damage, comorbidities, and demographic characteristics. A multivariable Cox regression model was built to calculate hazard ratios (HRs) for the first infection.

Results A total of 353 jSLE patients were included: $88.7 \%$ female, median age at diagnosis: 14.3 years (SD 2.9), and mean disease duration: 16.0 years (SD 9.3). A total of 104 $(29.5 \%)$ patients suffered 1 serious infection (1: 55.8\%; 2-5: $38.4 \%$, and 6 infections: $5.8 \%$ ). Sociodemographic data is shown in table 1.

Total serious infections recorded in these patients numbered 205. The incidence rate was 3.7 (95\% CI: 3.24.2) infections per 100 patient years.

In the bivariate analysis we found association between serious infections and smoking $(p=0.018)$, lupus nephritis $(\mathrm{p}<0.001)$, kidney transplantation $(\mathrm{p}=0.017)$, corticosteroids use $(p=0.02)$, higher corticosteroids dosage $(p<0.001)$, immunosupressants use $(\mathrm{p}<0.001)$ - azathioprine, mycophenolate, cychlophosphamide and rituximab, hospitalization due to jSLE flare $(p<0.001)$, higher SLEDAI score $(p=0.026)$, higher KATZ score $(\mathrm{p}<0.001)$ and higher CHARLSON score $(\mathrm{p}=0.02)$.

Serious infection localization and causal agent are described in table 1 , being respiratory and bacterial infections the most frequent, respectively.

In the logistic regression analysis the use of cyclophosphamide, mycophenolate and rituximab and SLICC score showed association to serious infection (OR 2,55 [1,44-4,52], OR 1,4 $[1,17-1,66]$, respectively; $\mathrm{p}<0.001)$. In the Cox regression

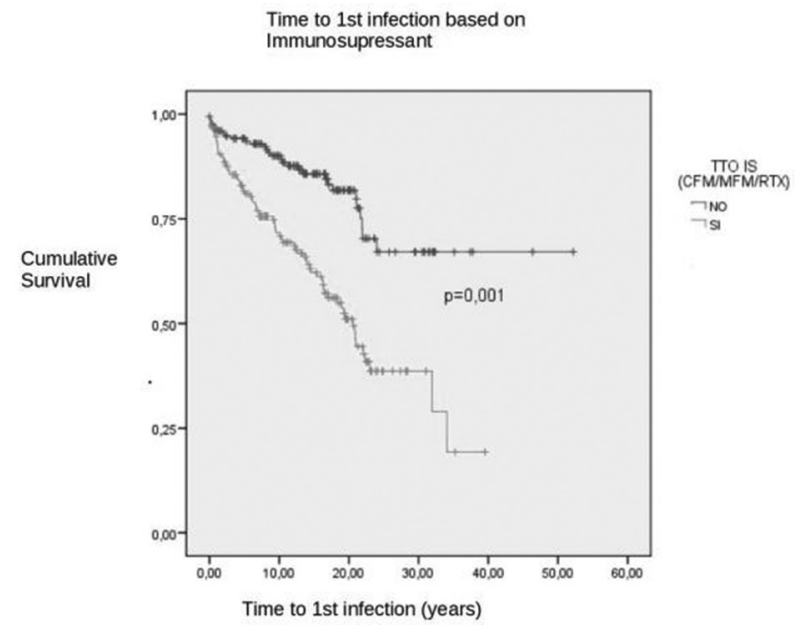

Abstract 181 Figure 1 Time to 1st infection based on immunosupressant treatment 
analysis, the following were all associated with serious infection $(p<0.01)$ : splenectomy, use of cyclophosphamide, mycophenolate and rituximab, which is shown in figure 1 and figure 2 .

Conclusions In the largest observational European Registry of SLE patients, one third of the jSLE patients suffered serious infections. Higher SLEDAI score, renal involvement and immunosupressant and corticosteroids use were independent associated factors to the presence of serious infection in jSLE, as well as smoking.

Funding Source(s): FIS Grant PI11/02857 (Instituto Carlos III, Fondos FEDER) has supported this work.

\section{IMMODULATORY MEDICATION USE FOR YOUTH WITH NEWLY-DIAGNOSED SYSTEMIC LUPUS ERYTHEMATOSUS}

${ }^{1}$ Alaina Davis, ${ }^{2}$ Andrea M Knight*, ${ }^{3}$ Marisa Klein-Gitelman, ${ }^{4}$ Jennifer Faerber, ${ }^{4}$ Hannah Katcoff, ${ }^{5}$ Zuleyha Cidav, ${ }^{5}$ David Mandell. ${ }^{1}$ Monroe Carell Junior Children's Hospital at Vanderbilt; ${ }^{2}$ The Hospital for Sick Children, Toronto; ${ }^{3}$ Ann and Robert $H$ Lurie Childrens Hospital of Chicago; ${ }^{4}$ The Children's Hospital of Philadelphia; ${ }^{5}$ University of Pennsylvania

\subsection{6/lupus-2019-Ism.182}

Background To examine immunomodulatory medication use for youth with systemic lupus erythematosus (SLE) during their first year of care.

Methods We conducted a retrospective cohort study using deidentified administrative claims for 2000 to 2013 from Optum $\left(\right.$ C Clinformatics ${ }^{\circledR}$ DataMart for youth ages $10-24$ years with an incident diagnosis of SLE (3 International Classication of Diseases, Ninth Revision codes for SLE 710.0, each $>30$ days apart). We determined the proportion of subjects filling a prescription for an immunomodulatory mediation, defined as hydroxychloroquine or an immunosuppressant (excluding glucocorticoids), within 3, 6, and 12 months after the first SLE diagnosis code (index date). We used a Cox proportional hazards regression model to examine associations between time to immunomodulatory prescription fill within 12 months and demographic and disease factors (age, race/ethnicity, household education level, region, history of seizures/stroke, history nephritis).

Results We identified 650 youth with an incident diagnosis of SLE. In the 12 months following the index date, 511 (79\%) of youth had a prescription fill for an immunomodulatory medication. For those with a prescription fill for hydroxychloroquine in the first year $(n=457,70 \%), 374(58 \%)$ and $407(63 \%)$ of youth filled the medication within 3 months and 6 months from the index date, respectively (table). For those with a prescription fill for an immunosuppressant $(n=221,34 \%)$ in the first year, $114(18 \%)$ and $162(25 \%)$ of youth filled the medication within 3 months and 6 months from the index date, respectively (Table). Location in the Northeast region was significantly associated with a longer time to immunomodulatory prescription fill within 12 months, compared to location in the South $(\mathrm{HR}=0.686$, 95\% CI 0.50 0.94). There were no statistically significant associations for the other demographic and disease factors.

Conclusions Among youth with newly-diagnosed SLE, hydroxychloroquine use is prevalent although not universal, and immunosuppressant use is notably low during the first year of care. As poorly controlled SLE disease activity can lead to organ damage, further work is needed to identify potential
Abstract 182 Table 1 Immunomodulatory Medication Use in Youth with Newly-Diagnosed SLE, N=650

\begin{tabular}{lccc}
\hline $\begin{array}{l}\text { Proportion with prescription } \\
\text { fills after first SLE diagnosis code, } \\
\mathbf{n}(\%)\end{array}$ & $\begin{array}{c}\text { Within } \\
3 \text { months }\end{array}$ & $\begin{array}{c}\text { Within } \\
6 \text { months }\end{array}$ & $\begin{array}{c}\text { Within } \\
1 \text { year }\end{array}$ \\
\hline $\begin{array}{l}\text { Immunomodulatory medication } \\
\text { (hydroxychloroquine or }\end{array}$ & $428(66)$ & $460(71)$ & $511(78)$ \\
$\begin{array}{l}\text { immunosuppressant) } \\
\text { Hydroxychloroquine }\end{array}$ & $374(58)$ & $407(63)$ & $457(70)$ \\
Immunosuppressant & $114(18)$ & $162(25)$ & $221(34)$
\end{tabular}

Immunosuppressant medications include: mycophenolate mofetil, azathioprine, leflunomide, methotrexate, tacrolimus, and oral cyclophosphamide.

factors contributing to suboptimal immunomodulatory medication use in this population.

Funding Source(s): The Childhood Arthritis and Rheumatology Research Alliance, Alpha Omicron Pi Foundation

\section{A PHASE 1B/2A TRIAL OF TOFACITINIB, AN ORAL JANUS KINASE INHIBITOR, IN SYSTEMIC LUPUS ERYTHEMATOSUS}

${ }^{1}$ Sarfaraz Hasni*, ${ }^{2}$ Sarthak Gupta, ${ }^{3}$ Michael A Davis, ${ }^{4}$ Elaine Poncio, ${ }^{5}$ Yenealem TemesgenOyelakin, ${ }^{6}$ Ann Biehl, ${ }^{7}$ Philip Carlucci, ${ }^{5}$ Xinghao Wang, ${ }^{8}$ Isabel Ochoa-Navas, ${ }^{8}$ Zerai G Manna, ${ }^{2}$ Mohammad Naqi, ${ }^{9}$ Yinghui Shi, ${ }^{10}$ Donald E Thomas, ${ }^{11}$ Jinguo Chen, ${ }^{12}$ Angelique Biancotto, ${ }^{13}$ Richard Apps, ${ }^{14}$ Foo Cheung, ${ }^{15}$ Yuri Kotiliarov, ${ }^{16}$ Ashley Babyak, ${ }^{17}$ Katie Stagliano, ${ }^{18}$ Wanxia Tsai, ${ }^{9}$ Laura Vian, ${ }^{19}$ Nathalia R Gazaniga, ${ }^{20}$ Valentina Giudice, ${ }^{21}$ Martin Playford, ${ }^{2}$ Stephen Brooks, ${ }^{5}$ Rishi R Goel, ${ }^{22}$ Meggan MacKay, ${ }^{23}$ Peter Gregersen, ${ }^{22}$ Betty Diamond, ${ }^{24}$ Xiaobai Li, ${ }^{25}$ Alan Remaley, ${ }^{26}$ Nehal Mehta, ${ }^{8}$ John O'Shea ${ }^{21}$ Massimo Gadina, ${ }^{27}$ Mariana J Kaplan. ${ }^{1}$ The Office of Clinical Director, National Institute of Arthritis and Musculoskeletal and Skin Diseases, National Institutes of Health; ${ }^{2}$ NIAMS/NIH; ${ }^{3}$ National Institute of Arthritis and Musculoskelatal and Skin Diseases, NIH; ${ }^{4}$ National Institute of Arthritis and Musculoskeletal and Skin Diseases, National Institutes of Health; ${ }^{5}$ NIH/NIAMS; ${ }^{6}$ National Institute of Arthritis and Musculoskeletal and Skin Diseases; ${ }^{7}$ New York University School of Medicine; ${ }^{8}$ NIAMS; ${ }^{9} \mathrm{NIH} ;{ }^{10}$ Arthritis and Pain Associates of PG County; ${ }^{11}$ NIAID; ${ }^{12}$ Sanofi; ${ }^{13}$ NIH Center for Human Immunology; ${ }^{14}$ The National Institutes of Health; ${ }^{15} \mathrm{CHI} / \mathrm{NIAID} / \mathrm{NIH} ;{ }^{16}$ National Institutes of Health, Clinical Center; ${ }^{17}$ Center for Human Immunology, NIH; ${ }^{18}$ National Institue of Arthritis and Musculoskleatal and Skin Diseases; ${ }^{19}$ The Scripps Research Institute; ${ }^{20}$ University of Salerno; ${ }^{21}$ National Institutes of Health; ${ }^{22}$ Feinstein Institute; ${ }^{23}$ Feinstein Institute For Medical Research; ${ }^{24}$ Clinical Center, $\mathrm{NIH} ;{ }^{25} \mathrm{NHLBI} ;{ }^{26}$ National Heart, Lung and Blood Institute; ${ }^{27}$ NIH NIAMS

\subsection{6/lupus-2019-Ism.183}

Background A pharmacologic intervention that modulates JAK/ STAT signaling pathways represents a novel approach for the treatment of Systemic Lupus Erythematosus (SLE). In animal models of SLE, tofacitinib improved clinical features, immune dysregulation and vascular dysfunction. The STAT4 risk allele is associated with higher risk of severe manifestations in SLE. We hypothesized that immune modulation in response to JAK/ STAT inhibition would be more robust in SLE subjects that carry the STAT4 risk allele.

Methods We conducted a phase $1 \mathrm{~b} / 2 \mathrm{a}$ randomized, doubleblind, placebo-controlled clinical trial of oral tofacitinib, $5 \mathrm{mg}$ twice daily, in 30 SLE subjects (2:1 drug to placebo ratio) with mild to moderate disease activity, stratified by the presence or absence of STAT4 risk allele. Study duration was 84 days (56 days of active treatment ; 28 days of off drug). In addition to recording adverse events (AEs), lipoprotein profile, non-invasive vascular function studies, immuno-phenotyping, and gene expression studies were performed. 